\title{
MUJERES ESCRIBIENDO RESISTENCIAS SOBRE EL MURO DE PALESTINA
}

\author{
WOMEN WRITING RESISTANCES ON THE WALL IN PALESTINE
}

Olga Blázquez Sánchez

Universidad Autónoma de Madrid

\section{RESUMEN:}

El muro que sigue siendo construido por el Gobierno deIsrael en Palestina seha convertido no sólo en una barrera fronteriza que dificulta el movimiento del pueblo palestino, sino también en una estructura arquitectónica que puede ser re-apropiada por la sociedad civil a través del arte como estrategia de resistencia. La mujeres juegan un rol fundamental en el desarrollo de esta forma de resistencia y su visibilidad como actores públicos es cada vez mayor, realizando reivindicaciones no sólo como palestinas sino también como mujeres.

\section{Palabras claves:}

Palestina, muro, grafiti, mujeres, resistencia.

\section{Abstract:}

The wall that is still being built by the Government of Israel in Palestine has not only become a frontier barrier that makes it difficult for Palestinian people to move, but also an architectural structure that can be artistically re-appropiated by civilians as a strategy of resistance. Women play a central role in the development of such a way of resistance and they are increasingly visible as public actors that make claims not only as Palestinians but also as women.

\section{KEY WORD:}

Palestine, wall, grafitti, women, resistance. 


\section{Introducción}

A través del presente artículo se pretende analizar el modo en el que las poblaciones y los elementos arquitectónicos que las rodean pasan a formar parte de un sistema de relaciones complejo, en el que se aprecian choques, intersecciones y también encuentros. Se parte de una perspectiva fenomenológica aplicada a la arquitectura, lo que supone dotar de importancia y relevancia a la capa más superficial, perceptible, cambiante y dinámica de las construcciones arquitectónicas, en lugar de partir de su estructura interna, inmutable y, generalmente, oculta. De este modo, se analizará el modo en el que una determinada construcción, el muro levantado por Israel para dividir territorios palestinos en Cisjordania de los asentamientos israelíes, se comporta en su contexto específico, así como las relaciones que se crean entre el muro y las poblaciones con las que cohabita. Al hablar de estas relaciones existentes entre la población y el muro, se hará especial hincapié en las formas de re-apropiación del muro desarrolladas por parte de mujeres palestinas a través de la escritura sobre la superficie de esta barrera en forma de grafitis. El objetivo final es dejar constancia del rol de la sociedad civil y, en especial, de las mujeres, y de su incidencia sobre el devenir político a través de actos tan aparentemente "insignificantes" como la escritura de palabras cobre un muro.

\section{Arquitectura, fenomenología y performatividad}

La confluencia entre la disciplina de la arquitectura y la perspectiva de la fenomenología ha hecho temblar los cimientos de la estructura y su carácter inmutable. Si lanzamos la mirada a través del prisma de la fenomenología, nos topamos de lleno con la capa más superficial y perceptible de la realidad, con lo que puede ser captado por los sentidos, con lo mutable y siempre cambiante. La fenomenología como movimiento filosófico atrajo de nuevo la atención sobre la realidad tal y como se nos presenta, sobre su manifestación evidente, restando importancia al estudio sobre los misterios ocultos que encierra la apariencia del mundo. Tal y como apunta el filósofo Mauriuce Marleau-Ponty en Phenomenology of Perception (1962), la fenomenología es "a philosophy for which the world is 'already there' before reflection begins" (vii), es decir, la fenomenología es una filosofía para la cual el mundo está 'ya ahi', antes de que comience la reflexión ${ }^{1}$. Desde el punto de vista arquitectónico, este giro hacia el fenómeno perceptible como fuente de conocimiento también ha inaugurado una vía para repensar el espacio, los edificios, las construcciones. Al centrar la atención sobre la superficialidad del mundo y su carácter cambiante, el espacio lejos de ser una categoría estática, se transforma en algo dinámico. El espacio está en movimiento. Bernard Tschumi, por ejemplo, en Architecture and Disjunction (1996), establece que no es posible el espacio sin evento (139). El espacio, lejos de ser un mero soporte o

1 Las traducciones tanto del inglés como del árabe al castellano son propias. recipiente de sucesos, se transforma en un suceso en sí mismo. El espacio es el evento, el espacio se hace, el espacio se ha transformado en una categoría performativa que actualiza constantemente su propia existencia. El espacio ya no es, el espacio está.

\section{Un muro en Palestina}

En 2002, el Gobierno de Israel aprobó la construcción ilegal de un muro con el objetivo de aislar las zonas israelíes de las zonas palestinas en Cisjordania bajo la excusa de defenderse de ataques terroristas. En la práctica, la construcción del muro ha servido de estrategia geopolítica, consumándose la anexión de determinados territorios palestinos por parte de Israel, así como de estrategia bélica y de desestabilización lanzada contra el pueblo palestino. Estructuralmente, el muro no es una construcción homogénea, sino que consta de diferentes secciones (vallado, alambradas, secciones de hormigón, puntos de control, etc.) que lo convierten en un mecanismo complejo que interfiere en la vida de las comunidades palestinas de muy variadas maneras.

Mirar hacia el muro construido en Palestina desde una perspectiva fenomenológica y performativa supone dotar a esta construcción de vida. El muro, así, pasa de ser algo inerte a ser un organismo cuya existencia interfiere de forma orgánica con las vidas de otros seres que circulan a través del mismo contexto. El muro de Palestina es un evento y goza de cierta independencia con respecto a los intereses y objetivos con los que está siendo construido. Qué duda cabe de que el muro cumple de forma absoluta la función con la que fue diseñado, es decir, dividir las áreas y los asentamientos israelíes de las zonas palestinas. Sin embargo, en el estrecho espacio que se encuentra entre la función con la que el muro fue programado o diseñado y la realidad palpable del muro, existe un lugar para la libertad, un lugar que no se puede programar. Y ese es el espacio que puede ser aprovechado para la resistencia.

Como se ha apuntado anteriormente, el espacio es performativo, lo que quiere decir que un espacio existe porque se da una ejecución o realización de la existencia a través de la repetición de determinados actos constituyentes. Judith Butler forjó el concepto de acto constituyente (constituing act) para reflexionar sobre la construcción performativa del género. Según Butler, en "Performative Acts and Gender Constitution: An Essay in Phenomenology and Feminist Theory" (1988), los actos constituyentes son aquellos actos que se repiten y terminan por constituir una identidad determinada. La identidad, pues, desde este punto de vista, se construye en el hacer, en el ejercicio de determinados actos. Si trasladamos esta reflexión efectuada por Butler con respecto al género al ámbito de la arquitectura, comprobaremos que un edificio también ejecuta determinados actos constituyentes, actos que dan cuerpo a su existencia y los cuales son necesarios para que podamos seguir afirmando que ese edificio es un edificio, y no otro tipo de construcción como puede ser un puente o un túnel. En el caso del 
muro de Palestina, se puede decir no sólo que el muro es un muro, sino también que es un mecanismo arquitectónico violento porque fue diseñado para ejecutar, y en la práctica ejecuta, violencia: impide la libre circulación de personas y el acceso a recursos necesarios como hospitales, escuelas, etc. Es cierto que, en cierta medida, como también apunta Tschumi, toda construcción arquitectónica es intrínsecamente violenta (Tschumi, 1996: 121), ya que interfiere en el movimiento de las personas e impone una estructura concreta al espacio. Sin embargo, en el caso del muro de Palestina, como ocurre con muchos otros casos de arquitectura de frontera, el quid de la cuestión radica en el que la violencia forma parte del diseño de la construcción. De forma general, cuando se diseña un edificio, no se piensa tanto en restringir el acceso de la población a un espacio concreto, como en la creación de un nuevo espacio y en facilitar su acceso a él. Puede que, a posteriori, el edificio sí que ejerza cierto grado de violencia sobre la población al impedir ciertos movimientos y trayectorias, sin embargo, ese no tiene por qué ser el objetivo al diseñarlo. Por el contrario, el caso del muro de Palestina es justamente el opuesto. Desde Israel se diseña específicamente un muro que ha de ser violento. Pero, ¿qué pasa cuando el muro de Palestina ya está construido? Cuando el muro de Palestina deja de ser un proyecto y se manifiesta de facto en la linde entre Israel y Palestina, se convierte directamente en un evento cuyo devenir deja de ser objeto exclusivo de las autoridades israelíes. El muro ejerce la violencia que de él se espera, pero también encarna la resistencia a esa violencia a través de la re-apropiación del muro por parte de las poblaciones que lo padecen. De este modo, el muro deja de pertenecer a Israel de forma exclusiva para convertirse en un espacio transitado y apropiado por la multiplicidad.

\section{El muro de separación en Palestina: una superficie para escribir y resistir}

A través de este estudio, se pretende profundizar en una forma específica de reapropiación del muro: el grafiti, y, en concreto, en los grafitis realizados por mujeres. A través del grafiti, observamos cómo el muro de Palestina se convierte en un especie de lienzo o papel, en una superficie sobre la que se inscriben y se escriben formas de resistencia. Sin embargo, esta superficie difiere en muchas de sus características de otras superficies empleadas para la escritura, como puede ser un folio o la página en blanco de un cuaderno. Pero, ¿cuáles son estas diferencias fundamentales? Más allá de las diferencias obvias entre, por ejemplo, un folio y el muro de Palestina (tamaño, material, resistencia, etc.) existen otras tres fundamentales:

En primer lugar, mientras que un folio o la página de un cuaderno son reversibles, es decir, se pueden emplear por ambos lados, en el caso del muro de Palestina existe un claro desequilibrio entre uno de los lados y el otro. Es cierto que ambos lados están prohibidos, sin embargo, el lado orientado hacia cualquier territorio palestino resulta más accesible que el situado en la parte israelí. No sólo se trata de una accesibilidad física (obviamente una persona palestina tiene mejor acceso a la parte del muro situada en el lugar en el que habita), sino que también entra en juego la tensión de poderes. El muro es una creación israelí y, por ende, ambas partes del mismo pertenecen a Israel. Ahora bien, un grafiti escrito en el lado israelí será considerado siempre como una provocación de mayor calibre o envergadura que un grafiti situado en la parte palestina, por lo que la vigilancia y estricto seguimiento de lo que pasa en una parte del muro y en la otra no será nunca el mismo. Incluso para un artista callejero israelí será siembre más fácil hacer una pintada o inscripción en el lado palestino que realizarlos en la parte israelí, como pone de manifiesto el artista afincado en Tel Aviv, Know Hope, cuya historia aparece retratada en el artículo de La Vanguardia titulado “La Paz de Grafiti" (2012).

En segundo lugar, el poder y el ejercicio del poder son claves para entender el carácter subversivo de la escritura en el caso del muro de Palestina. Cuando se escribe sobre un folio, el acto mismo de escribir no se convierte necesariamente en un acto subversivo. El carácter subversivo o no de la escritura, en este caso, depende más del contenido, de lo que se dice a través de la escritura. Por el contrario, en el caso del muro de Palestina, el acto mismo de escribir se convierte en subversión y resistencia puesto que supone una utilización prohibida del muro. Por otra parte, escribir sobre un folio es casi siempre un acto privado que, posteriormente, puede hacerse público o no. Pero las inscripciones sobre el muro de Palestina son siembre un acto público.

Finalmente, mientras que el acto de escribir sobre un folio no supone una transformación del objeto-folio, ya que la función con la que el folio fue diseñado y su uso están en perfecta consonancia, en el caso de las inscripciones sobre el muro sí se observa una metamorfosis. Un folio escrito no deja de ser un folio, aunque su apariencia se haya visto modificada. Incluso aunque el folio no se empleara para escribir, sino, por ejemplo, para hacer papiroflexia, el folio no perdería por completo su identidad, ya que no hay una prohibición explícita con respecto al uso que se puede hacer o no de una hoja en blanco. En el caso del muro de Palestina esta prohibición es evidente, por lo que el acto de escribir o pintar sobre él supone una transformación del espacio. Un muro que había sido concebido exclusivamente para separar y reprimir el movimiento, se convierte, al escribir, en un espacio de expresión y de canalización de movimiento de resistencia al mismo.

\section{Mujeres ESCRIBIENDO SOBRE EL MURO}

¿Y qué hay de las personas que escriben sobre el muro? De todas las personas que escriben y/o pintan sobre el muro de Palestina, la mayoría siguen siendo hombres. $Y$ de todos los hombres y mujeres que escriben y/o pintan sobre el muro de Palestina, la 
mayoría siguen siendo extranjeros/as, como apunta la artista palestina Areej Mawasi, cuyas palabras fueron recogidas en la entrada del blog suzeeinthecity titulada "Graffiti in Palestine: Female Street Artist from East Jerusalem and Rockets over Gaza", publicada el 15 de Noviembre de 2012. Como prueba de este desequilibrio existente entre el protagonismo de artistas internacionales y locales, basta simplemente contemplar los resultados de búsqueda que arroja Google al teclear conjuntamente las palabras "muro", "Palestina" y "grafiti". Gran parte de estos resultados se refieren a grafitis realizados por el artista británico Banksy. En cuanto a las mujeres que acuden al muro para dejar su huella sobre él, cabe destacar también el protagonismo que cobran las que forman parte de campañas internacionales de apoyo a la causa palestina. Éste fue el caso de las artistas españolas Den, Musa, Yubia, Mamba y Mako, que, como finalistas del el I Certamen Internacional de Mujeres Artistas "Graffiti por la Paz", celebrado en diciembre de 2010, fueron galardonadas con la oportunidad de realizar sus pintadas sobre el muro que divide la ciudad de Belén. De esta situación, marcada por el escaso, aunque creciente, protagonismo de las mujeres palestinas en los actos de resistencia, se deriva el hecho de que los grafitis realizados por ellas, generalmente, no se circunscriban a un único marco de lucha, sino a varios. Es decir, las mujeres palestinas, a través de los grafitis realizados sobre el muro, no sólo reclaman derechos para el pueblo palestino o denuncian los abusos cometidos por Israel, sino que también reflejan y canalizan, a través de sus escritos y pinturas, la lucha contra el patriarcado y la necesidad de convertirse en protagonistas de sus propias luchas a través de la conquista del espacio público. Así, Laila, pseudónimo utilizado por una de las mujeres que forman parte de un colectivo de activistas en Jerusalén, en el artículo "Taking back Palestine's streets: excusive interview with underground Jerusalem graffiti artist", publicado en The Electrnic Intifada (2012) subraya la importancia de la lucha feminista de las mujeres palestinas, y justifica, de este modo, su propia práctica artística, en la que, a través de sus grafitis, hace un llamamiento no tanto a las autoridades israelíes para que cesen en su ahogo al pueblo palestino, como a las mujeres palestinas para que se alcen en contra del carácter patriarcal de su propia sociedad, paso necesario para conseguir la liberación del pueblo palestino en su totalidad.

Desde una perspectiva menos marcadamente política (aunque también lo sea), se percibe el trabajo de Areej Mawasi. Areej Mawasi es una artista palestina afincada en Jerusalén que, sirviéndose de una plantilla, realiza inscripciones sobre las paredes de la ciudad, no solamente sobre el muro de Palestina. Esta artista palestina comenzó realizando inscripciones en su barrio hasta que, con el tiempo, sus pintadas fueron ganado visibilidad y protagonismo. Una de las inscripciones más famosas que ha realizado es aquella en la que se puede leer ๑® ๑๐®), cuya transcripción es fy amal, y que se puede traducir como hay esperanza. Resulta interesante pensar en el nivel de expresividad que alcanzan estas palabras más allá de la propia autoría de las mismas, así como de la dimensión política que alcanzan más allá de su significado más primario, $\mathrm{y}$, al menos aparentemente, apolítico. ¿Hay esperanza en una zona de conflicto que parece no encontrar la salida? El mensaje mismo de Areej Mawasi se transforma en una expresión irónica, mordaz y sarcástica, pero también en un deseo casi utópico y nostálgico. Las palabras inscritas sobre una pared son lo que son y también su opuesto. Hay esperanza se convierte en un letanía que se repite en un contexto de continua represión. Hay esperanza a pesar de todo. Hay esperanza aunque no haya esperanza. Las palabras y su soporte (el muro y otras paredes de Jerusalén) entran en un juego dialéctico, en un baile en el que el significado y su calado político, aparecen y desaparecen, dependiendo del momento y dependiendo, incluso, del estado de la persona que lea dichas palabras.

\section{Mujeres representadas sobre el muro}

Además de ser escritoras de sus propias luchas, las mujeres palestinas también son representadas sobre el muro de Palestina. Quizás el ejemplo más paradigmático lo constituya Leila Khaled, símbolo de la lucha palestina. Khaled, miembro del Frente Popular para la Liberación de Palestina, terminó de forjarse como un icono de la resistencia tras los sucesos del 29 de agosto de 1969, fecha en la que se convirtió en la primera mujer palestina que secuestraba un avión de pasajeros, el cual realizaba el trayecto Roma - Atenas. Khaled desvió el avión hacia Damasco y destruyó la nave tras el aterrizaje sin causar víctimas mortales. El rostro de esta mujer fue posteriormente fotografiado, difundido y también representado sobre el muro, pasando a formar parte de la imaginería de la resistencia palestina. El rostro de Khaled aparece en los grafitis generalmente acompañado de textos como "I am not a terrorist", no soy una terrorista o "don't forget the struggle", no olvides la lucha, a través de los cuales se pone de manifiesto la discusión que sobre esta mujer se lleva desarrollando durante años: ¿sus acciones deben ser leídas como actos de terrorismo o como actos de resistencia?

Por otro lado, mujeres anónimas también son representadas sobre los muros de Palestina. Se encuentran ejemplos como las pintadas de Banksy, que suele acudir a la imagen de la niña cuando quiere retratar la inocencia y el carácter inofensivo del pueblo palestino, además de sus deseos de libertad. Este es el caso de dos de los más famosos grafitis del artista británico: el de una niña rebasando la barrera de Cisjordania gracias a unos globos y el de una niña cacheando a un soldado sobre el muro. Sin embargo, en este caso, no es la mujer palestina la que está siendo representada, y se podría decir, incluso, que la imagen de la niña, tal y como la presenta Banksy, traslada la imaginación hacia un contexto más occidental. Es decir, tal y como Mawasi denunciaba al referirse a la situación de las autoras de grafiti, en el caso de las mujeres representadas sobre el muro se aprecia también una marcada ausencia de protagonismo de las propias mujeres 
palestinas. Por otra parte, el carácter patriarcal tanto de las sociedades occidentales, cuyos embajadores/as artísticos en forma de grafiteros y grafiteras suelen retratar a las mujeres palestinas atendiendo a clichés reduccionistas, así como el carácter patriarcal de la sociedad palestina, influye en el modo en el que se representa a las mujeres palestinas que aparecen sobre el muro. Siguen proliferando las imágenes de madres, como en el caso de una de las pintadas situadas en el muro del campo de refugiados de Qalandiya, en la que aparece una mujer con hijab sosteniendo a un bebé. No obstante, también se percibe un cambio en el discurso de la resistencia palestina a través del grafiti, que reconoce cada vez más abiertamente la importancia del protagonismo de las mujeres en la lucha por la liberación. Reflejo de ello son las pintadas que un grupo de grafiteros realizaron en 2012 en pleno corazón de Jerusalén Oeste, entre las que destacaban las representaciones de la cara de una mujer palestina cubierta con la kufiyya y acompañada de palabras como intifada o revolucionario (๑๐๐)).

\section{Conclusión}

El acto, casi invisible, de escribir sobre una estructura arquitectónica megalítica como es el muro de Palestina no supone un duro golpe sobre las políticas violentas desarrolladas por el gobierno de Israel. Sin embrago, como apunta Laila en el reportaje recogido por The Electronic Intifada, sí sirve para crear "a system of resistance", un sistema de resistencia, en el que cada acto, cada pintada y cada inscripción es "part of a larger web of popular resistance", parte de una red mayor de resistencia popular. La relevancia e importancia de las acciones consistentes en realizar pintadas y escribir palabras sobre el muro, pues, radica en su capacidad, por pequeña que sea, para seguir tejiendo y entretejiendo lianas que sirvan para la canalización y la articulación de cuestiones sociales y políticas. Además, todas estas acciones también contribuyen a la interconexión de diferentes actores sociales, tendiendo puentes de encuentro para la discusión y la acción dentro de un marco más amplio de movilización sociopolítica.

\section{REFERENCIAS BIBLIOGRÁFICAS}

Butler, J., "Performative Acts and Gender Constitution: An Essay in Phenomenology and Feminist Theory", Theatre Journal, 40-4 (1988), pp. 519-531.

Chavarría, M., “La PazdeGrafiti”, La Vanguardia.Internet.1-04-2012.<http://www.lavanguardia. com/cultura/ 20120401/54279467444/arte-callejero-israeli-paz-del-grafiti.html>

Marleau-Ponty, M., Phenomenology of Perception, London, Routledge and Kegan Paul Ltd, 1962. Morayef, S., "Graffiti in Palestine: Female Street Artist from East Jerusalem and Rockets over Gaza", Suzeeinthecity. Internet. 15-11-2012. <https://suzeeinthecity.wordpress. com/2012/11/15/graffiti-in-east-jerusalem-female-street-artist-from-palestine-rockets-overgaza/>
Musleh, M., “Taking back Palestine's streets: exclusive interview with underground Jerusalem graffiti artist", The Electronic Intifada. Internet. 29-08-2012. https://electronicintifada.net/ content/taking-back-palestines-streets-exclusive-interview-underground-jerusalemgraffiti-artist

Nuevatribuna.es, "Artistas españolas llenan de colores el Muro", Nuevatribuna.es. Internet. 23-12-2010. < http://www.nuevatribuna.es/articulo/sociedad/artistas-espaolas-llenan-decolores-el-muro/ 20101228040353039484.html>

Tschumi, B., Architecture and Disjunction, London, MIT Press, 1996. 